\title{
National reporting system for medical errors is launched
}

Vittal Katikireddi $B M J$

The world's first national system for collecting reports of health system failures and any error that compromises patients' safety was launched this week.

The national reporting and learning system (NRLS), which aims to improve patients' safety by collecting reports from health professionals across England and Wales, has been developed by the National Patient Safety Agency (NPSA), an organisation established in 2001 to tackle the problem of errors in the NHS

The system will mostly extract information from existing local risk management systems. However, NHS health professionals will be able to report directly to the system through an online form if no local risk management system exists or if they prefer to do it that way. The system will retain information only in an anonymous form, and individual staff or patients involved in any incident will not be identified, says the agency.

The health minister Norman Warner launched the initiative at the agency's conference in Birmingham this week. He said: "It is essential that such incidents are reported locally, investigated, and analysed so that suitable learning and actions can follow. At the national level the NRLS will enable the NPSA to take an unprecedented overview, identify recurring patterns, and then develop practical solutions that can be applied consistently at the local level."

Susan Williams, who with Sue Osborn is joint chief executive of the agency, said: "Every day more than a million people are treated safely in the NHS However, evidence tells us that in complex healthcare systems some things will go wrong. Research has also shown that errors fall into recurrent patterns regardless of the people involved.

"In developing the NRLS we have drawn from the experience of other sectors, such as the aviation industry, which shows clearly that as reporting levels rise the number of serious errors begins to decline."

The agency is planning to publish statistics on trends and issues that are identified through the system to promote a "learning culture" in the NHS. Ms Williams said: "Based on our consultations, health trusts and hospitals are quite keen on receiving comparative information to help them improve.

"We would not publish ratings of hospitals or trusts. However, we're working with the new Commission for Healthcare Audit and Inspection to help them make a judgment about how hospitals and trusts are doing with regard to patient safety.

"Although it seems counterintuitive, what we want to see is an increasing awareness of safety issues so that the number of reports increase but the incidence of serious harm goes down. In that sense we're hoping to mirror the experience of many other industries that we've looked at, including the aviation, railway, maritime, and nuclear industries."

The system was initially piloted in 28 trusts across England and Wales in 2001. After substantial problems with the pilot (BMJ 2002;324:1473) the system was further tested in 39 trusts.
The system is expected to cost about $£ 1 \mathrm{~m}(\$ 1.9 \mathrm{~m} ; € 1.5 \mathrm{~m})$ a year for the first eight years. "But we're not expecting it to cost as much as that in the later years," Ms Williams added.

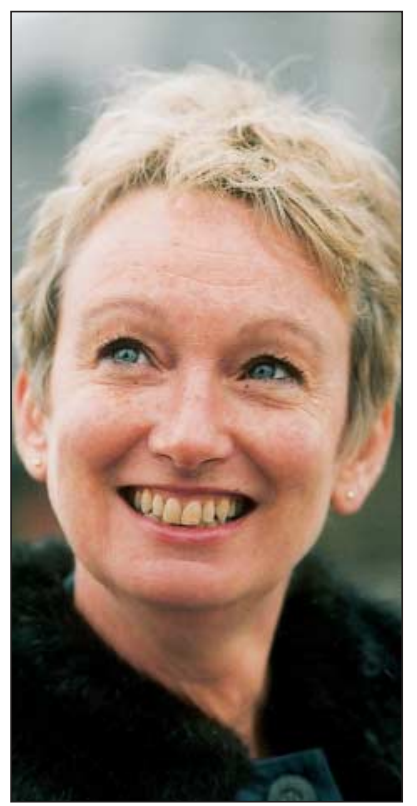

Susan Williams: "As reporting levels rise the number of serious errors begins to decline"

\section{Emergency team phone numbers should be standardised}

Vittal Katikireddi $B M J$

The phone numbers used to call a hospital's emergency care team should be standardised across all NHS acute trusts in England and Wales, Lord Hunt, chairman of the National Patient Safety Association, said at the association's conference this week.

Lord Hunt has advised NHS hospitals to use the number 2222 in future, after the association found that at least 27 different "crash call" numbers are currently in use in England and Wales.

The health minister Lord Warner said: "This is another step in the right direction towards improving the quality of care patients receive. Having one number to summon emergency care will minimise confusion for staff and mean that patients get the treatment they need, fast. It is part of a wider drive to reduce unnecessary bureaucracy on the front line so that staff can get on with the job of caring for patients."

The initiative has received wide support from professional organisations.

"Many NHS staff, particularly junior doctors, frequently move between hospitals," said Simon Eccles, chairman of the BMA's Junior Doctors Committee. "Standardising the crash call number is a commonsense measure that will improve patient safety."

John Hayworth, chairman of the British Association for Accident and Emergency Medicine and a consultant at Southampton General Hospital, welcomed the initiative. "The idea of having different numbers in different hospitals seems to be nonsense. Many staff, including doctors, nurses, and other health professionals, move from hospital to hospital; so having a standard number, which would alert the crash team, is a welcome and indeed overdue move," he said.

The National Patient Safety Association estimates that the average cost for trusts of converting to 2222 will be $£ 5200$ (\$9700; €7730). This includes changes to switchboard technology and informing staff of the change through training, new signs, and emergency directories. 